\title{
Sarcoidosis misdiagnosed as malignant tumors: a case report
}

\author{
Zuosheng $\mathrm{Li}^{1,2+}$, Xin $\mathrm{Li}^{1 \dagger}$, Zuoqing Song ${ }^{1}$, Jinghao Liu', Ming Dong ${ }^{1}$, Tao Shi ${ }^{1}$, Dian Ren ${ }^{1}$, Song X ${ }^{1 *}$ \\ and Jun Chen ${ }^{1 *}$
}

\begin{abstract}
Background: Sarcoidosis is a rare condition that is often misdiagnosed as malignant tumors due to the similar clinical manifestations and imaging findings.

Case Presentation: We encountered a 56-year-old Chinese woman who had a chief complaint of a persistent cough. The chest computer tomography (CT) revealed mediastinal and bilateral hilar lymph node enlargement, and positron emission tomography-computer tomography (PET-CT) revealed abnormal fluorodeoxyglucose (FDG) uptake in the lymph nodes of the chest and abdomen. To further clarify the diagnosis, a lymph node sampling was performed by video-assisted thoracoscopic surgery (VATS) and the histopathologic diagnosis of sarcoidosis was confirmed.
\end{abstract}

Conclusions: VATS could be an effective and minimally invasive diagnostic method to discriminate pulmonary sarcoidosis with other malignant tumors.

Keywords: Sarcoidosis, Lung cancer, Differential diagnosis

\section{Background}

Sarcoidosis is an uncommon disease of unknown etiology that is characterized by the presence of noncaseating granulomas [1]. Sarcoidosis affects people of all racial and ethnic groups and occurs at all ages, although it usually develops before the age of 50 years, with the incidence peaking at 20 to 39 years [2]. Sarcoidal granulomas can involve any organ, but in more than $90 \%$ of patients, clinical sarcoidosis is manifested as intrathoracic lymph node enlargement, pulmonary involvement, skin or ocular signs and symptoms, or some combination of these findings [3]. Systemic symptoms such as fatigue, night sweats, and weight loss are common. Sarcoidosis patients may be misdiagnosed with tuberculosis, lymphoma, or lung cancer [4]. The diagnosis of sarcoidosis is established on the basis of compatible clinical and radiologic findings, supported by histologic evidence in one or more organs of noncaseating epithelioid-cell granulomas in the absence of organisms or particles.

\footnotetext{
* Correspondence: xusong198@hotmail.com; huntercj2004@yahoo.com ${ }^{\dagger}$ Equal contributors

${ }^{1}$ Department of Lung Cancer Surgery, Lung Cancer Institute, Tianjin Medical University General Hospital, No. 154 Anshan Road, Heping District, Tianjin 300052, China

Full list of author information is available at the end of the article
}

\section{Case presentation}

A 56-year-old Chinese woman was admitted with cough and expectoration (white phlegm) for 1 month. These symptoms developed without apparent cause and in the absence of other problems, such as chest pain and/or distress, breathlessness, fever, hemoptysis, nausea, or vomiting. She denied the presence of any chronic disorders and smoking and had no family history of cancers. An enhanced chest computed tomography (CT) scan imaged a right hilar mass and a small amount of bilateral pleural effusions, accompanied with the enlargement of multiple lymph nodes in the mediastinal and bilateral hilar areas, as shown in Fig. 1. The blood tests and urinalysis on admission were within normal limits. To assist the diagnosis of the mass, a panel of tumor biomarkers, including CEA (carcino-embryonic antigen), NSE (neuron-specific enolase), SCC (squamous cell carcinoma), and CYFRA21-1 (cytokeratin 19 fragments), were tested. Only an upregulated level of NSE was observed (19.94 $\mu \mathrm{g} / \mathrm{L}$, normal reference value 0$16.3 \mu \mathrm{g} / \mathrm{L})$. Bronchoscopic exam did not reveal any abnormality, and a transbronchial biopsy by endobronchial ultrasound guided transbronchial needle aspiration (EBUS-TBNA) did not found collections of malignant cells either. A body fluorodeoxyglucose 


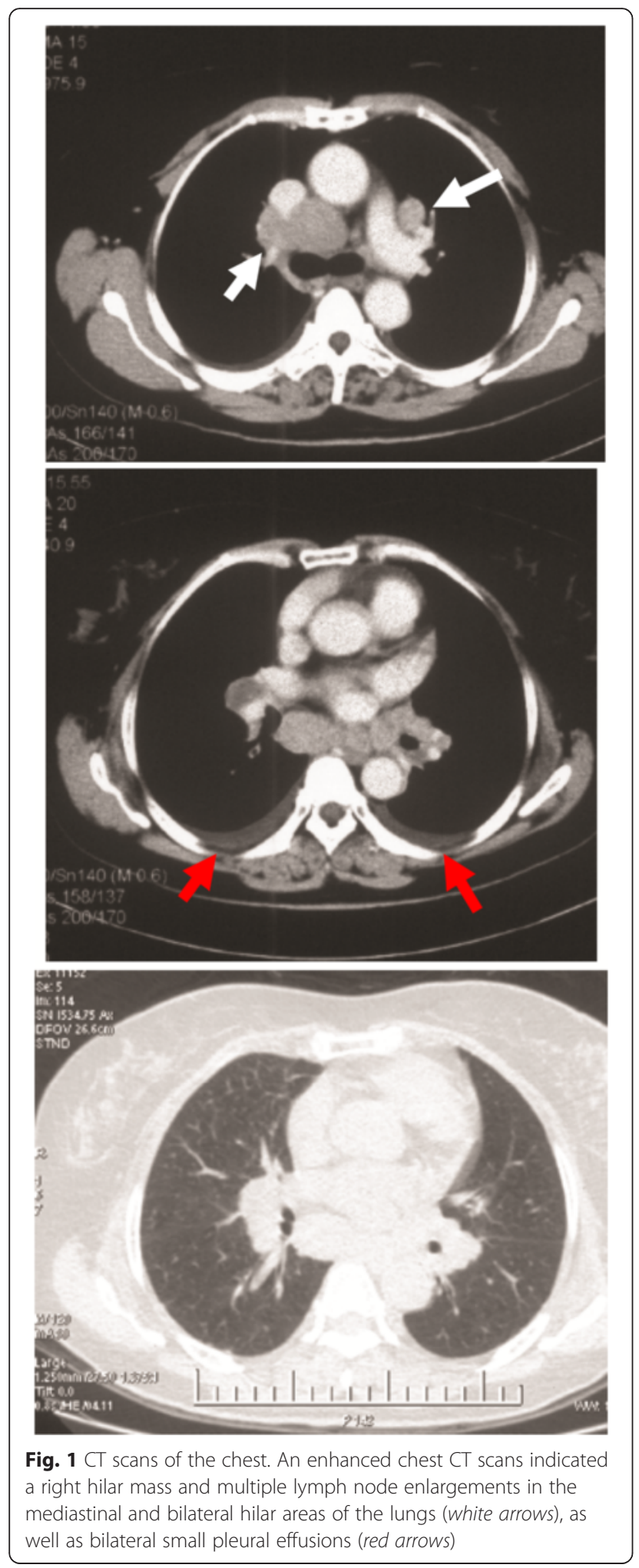

(FDG) positron emission tomography-computer tomography (PET-CT) demonstrated remarkably increased FDG uptake in the hilus of the right lung, accompanied by multiple lymph node enlargements in bilateral hilar areas, mediastinum, and abdomen (Fig. 2). Therefore, the diagnosis of malignant tumors was preliminarily made.

To further clarify the diagnosis, a subcarinal lymph node resection was performed by right-sided videoassisted thoracoscopic (VATS). In brief, a 40-mm incision was made in the fifth intercostal space of the right anterior axillary line for the instrumental operation. A 30-degree $10 \mathrm{~mm}$ high definition thoracoscope was employed through the seventh intercostal space of the right mid-axillary line. It was observed from the thoracoscope that mediastinal lymph nodes were diffusely enlarged, and lymph node biopsy was applied by a harmonic scalpel. As shown in Fig. 3, histopathologic examination was granulomatous lesions and a diagnosis of sarcoidosis was confirmed. After the postoperative recovery, the patient was transferred to the respiratory department to receive further medication treatment.

\section{Discussion}

Sarcoidosis is a multisystemic disorder of unknown etiology that is characterized by the presence of noncaseating granulomas and the proliferation of epithelioid cells [1]. Sarcoidosis can involve multiple organs; the lungs and lymph node are the most frequently involved organs. The cause and pathogenesis are unknown; however, a number of etiologies have been proposed in recent years, primarily based on genetic and immunologic factors [5]. The diagnosis of sarcoidosis is commonly established based on clinical and radiologic findings that are supported by histologic findings. The diagnosis of sarcoidosis is often delayed for various reasons, mainly due that the imaging is not suggestive sometimes. A study from Brazil evaluated 100 patients with a biopsy-proven diagnosis of sarcoidosis. The median number of physicians consulted was 3 (range, 114), and the diagnosis of sarcoidosis was timely in 41 patients and delayed in 59 [6]. The typical radiologic findings associated with sarcoidosis include symmetric, bilateral hilar, and paratracheal lymphadenopathy, with or without concomitant parenchymal abnormalities. However, in $25-30 \%$ of cases, the radiologic findings are atypical, which causes difficulty in making a correct diagnosis [7]. Atypical forms of intrathoracic sarcoidosis have been described as unilateral or asymmetric lymphadenopathy, necrosis or cavitation, large opacity, ground glass opacity, airway abnormality, pleural involvement, and the reversed halo sign [7-9]. A better understanding of the radiologic manifestations of sarcoidosis is very helpful for making a correct diagnosis. In addition, we observed that NSE, a well-known tumor marker for neuroendocrine tumors, was upregulated a bit in this case. Given the radiological image and the upregulated value of NSE, the diagnosis of small cell lung cancer was ever considered before the biopsy of VATS. However, the 

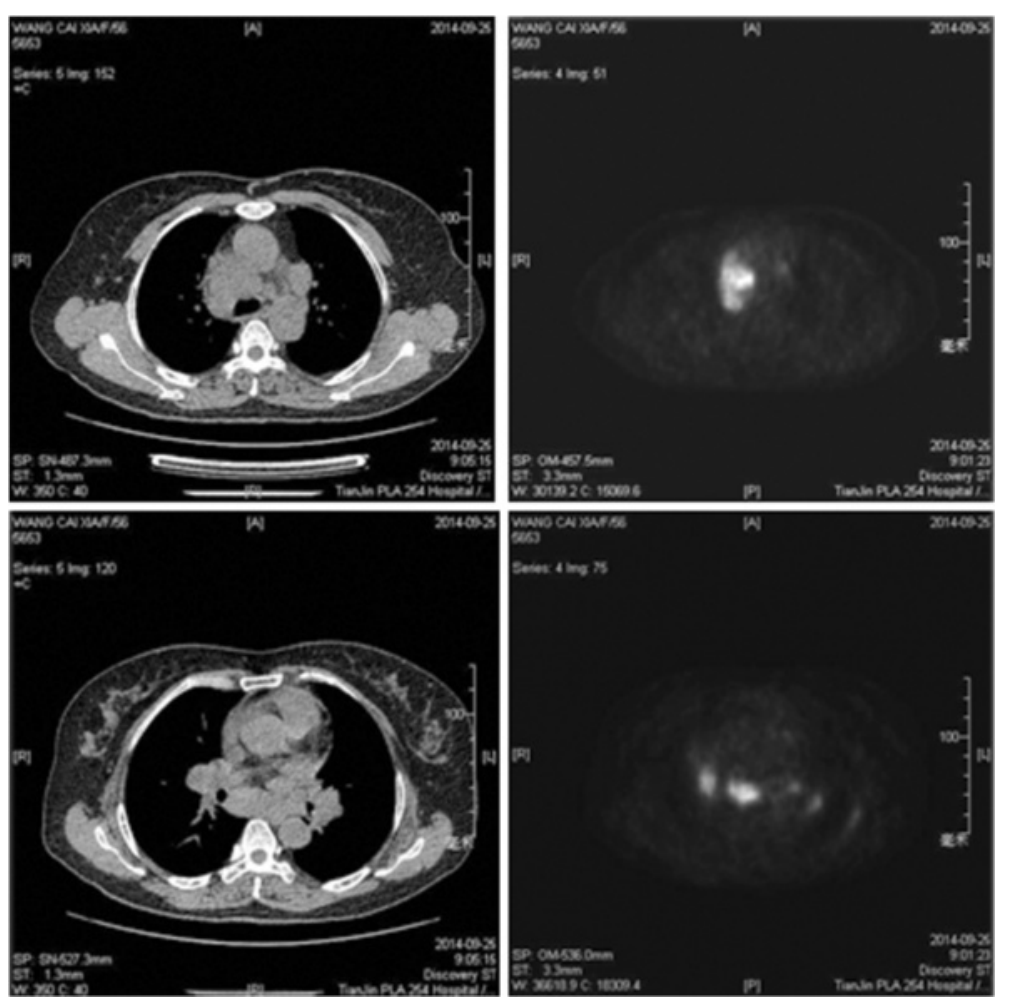

Fig. 2 PET-CT scan. PET-CT revealed abnormal FDG uptake in the lymph nodes of the upper mediastinum and bilateral hilar areas of the lungs

final pathological diagnosis did not support our speculation, indicating that sarcoidosis may be misdiagnosed with other malignant tumors if only tumor markers were considered.

In recent years, PET-CT has become a superior means of diagnosis of sarcoidosis. Moreover, ${ }^{18}$ F-FDG PET has been proven to be a very useful imaging technique in diagnosis, disease activity assessment, monitoring treatment response, and risk assessment in patients with sarcoidosis [10]. In suspected or known sarcoidosis patients, PET-CT may be useful in the evaluation of disease extent and monitoring treatment response [11]. In atypical, complex, and multisystem sarcoidosis, PET-CT plays an important role for the evaluation of the localization and effect of treatment [12]. Although it has so many advantages, PET-CT can cause many falsepositive and its role in the diagnosis of sarcoidosis is limited [13]. Pleural effusion is rare in patients with pulmonary sarcoidosis. Only about $2.8-5 \%$ of these patients exhibit pleural effusion [14-16]. A pleural effusion in patients with sarcoidosis should not be assumed to be related to sarcoidosis [16]. Pleural effusion in sarcoidosis is often an exudate. The most common cause of exudative pleural effusion in elderly patients is a malignant tumor or tuberculosis. Sarcoidosis is hard to be differentiated with the two diseases. In this patient, the CT scan showed a right hilar mass and a small amount of bilateral pleural effusions, accompanied with the enlargement of multiple lymph nodes in the mediastinal and bilateral hilar areas, while PET-CT indicated a high FDG uptake

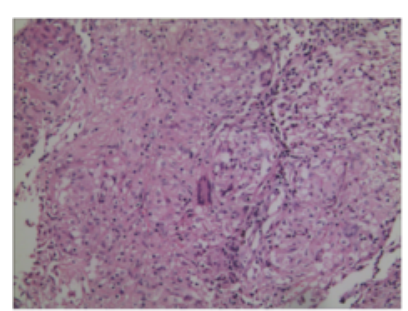

$\mathrm{x} 100$

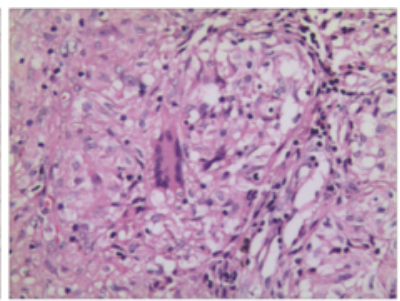

$\mathrm{x} 200$

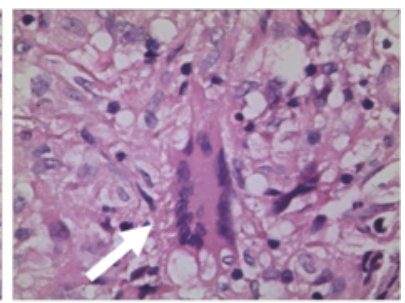

$\mathrm{x} 400$

Fig. 3 Histopathological diagnosis. The routine H\&E staining proved a diagnosis of sarcoidosis with granuloma plus Langhans giant cells (white arrows) 
in these lesions. The pathological diagnosis of sarcoidosis was determined after a video-assisted thoracoscopic procedure. Timely and correct diagnosis is very important for improving pulmonary function and determining the presence of lesions in other organs, while delayed diagnosis is often associated with an impaired lung function and medication induced impairment of the other organs [6]. For example, many sarcoidosis patients are misdiagnosed with tuberculosis and treated with antituberculosis drugs who eventually developed with liver function impairment. In addition, some serious forms of sarcoidosis, such as cardiac sarcoidosis, can appear undetected because of the lack of systematic investigation [6]. For these reasons, the diagnosis of sarcoidosis should be promptly determined in order to avoid treatment delay. Surgical intervention is an important modality for the attainment of a biopsy. VATS could be used as a valid minimally invasive method for the diagnosis of sarcoidosis, as it allows larger samples of tissue and greater diagnostic yield. Furthermore, in comparison with the traditional open techniques, VATS offers less postoperative pain, a more rapid recovery, and much less complications $[17,18]$.

\section{Conclusions}

In conclusion, sarcoidosis is difficult to be differentiated with other malignant tumors by radiological and PETCT examinations. VATS biopsy is a minimally invasive, safe, and effective procedure in the diagnosis of pulmonary sarcoidosis.

\section{Consent}

The patient granted written informed consent for publication of this manuscript and the accompanying images. A copy of the written consent is available for review by the Editor-in-Chief of this journal.

\section{Institutional review board statement}

This material has not been published and is not under consideration elsewhere. There is no financial disclosure from each author.

\section{Competing interests}

The authors declare that they have no competing interests.

\section{Authors' contributions}

$\mathrm{ZSL}$ and $\mathrm{XL}$ collected all the data and authored the manuscript. ZQS, JHL, $M D$, and DR were responsible for the patient care and analysis of follow-up data. TS prepared the histopathology. SX and JC performed the surgical procedure and also contributed to the data analysis and shaping of the manuscript. All authors have read and approved the final manuscript.

\section{Acknowledgements}

This research was supported by the grants from the National Natural Science Foundation of China (81172233 to Jun Chen, 81301812 to Song Xu),

Specialized Research Fund for the Doctoral Program of Higher Education (20131202110004 to Jun Chen, 20131202120004 to Song Xu), Scientific

Research Foundation for the Returned Overseas Chinese Scholars of State
Education Ministry (to Song Xu), Tianjin Science and Technology Support Program (12ZCDZSY16100 to Jun Chen), and Tianjin Educational Committee Foundation (20120117 to Song Xu).

\section{Author details}

'Department of Lung Cancer Surgery, Lung Cancer Institute, Tianjin Medical University General Hospital, No. 154 Anshan Road, Heping District, Tianjin 300052, China. ${ }^{2}$ Department of Thoracic Surgery, North China University of Science and Technology Affiliated Hospital, Tangshan 063000, China.

Received: 21 August 2015 Accepted: 7 December 2015

Published online: 12 December 2015

\section{References}

1. Colby TV. Pulmonary pathology in patients with systemic autoimmune diseases. Clin Chest Med. 1998;19(4):587-612. vii.

2. Rybicki BA, Major M, Popovich Jr J, Maliarik MJ, lannuzzi MC. Racial differences in sarcoidosis incidence: a 5-year study in a health maintenance organization. Am J Epidemiol. 1997;145(3):234-41.

3. Iannuzzi MC, Rybicki BA, Teirstein AS. Sarcoidosis. N Engl J Med. 2007;357(21):2153-65.

4. Hawtin KE, Roddie ME, Mauri FA, Copley SJ. Pulmonary sarcoidosis: the 'great pretender'. Clin Radiol. 2010;65(8):642-50.

5. Chen ES, Moller DR. Sarcoidosis-scientific progress and clinical challenges. Nat Rev Rheumatol. 2011;7(8):457-67.

6. Rodrigues MM, Coletta ENAM, Ferreira RG, Pereira CAD. Delayed diagnosis of sarcoidosis is common in Brazil. J Bras Pneumol. 2013;39(5):539-46.

7. Park HJ, Jung Jl, Chung MH, Song SW, Kim HL, Balk JH, et al. Typical and atypical manifestations of intrathoracic sarcoidosis. Korean J Radiol. 2009:10(6):623-31.

8. Kumazoe H, Matsunaga K, Nagata N, Komori M, Wakamatsu K, Kajiki A, et al. "Reversed halo sign" of high-resolution computed tomography in pulmonary sarcoidosis. J Thorac Imaging. 2009;24(1):66-8.

9. Marchiori E, Zanetti G, Mano CM, Hochhegger B, Irion KL. The reversed halo sign: another atypical manifestation of sarcoidosis. Korean J Radiol. 2010;11(2):251-2.

10. Skali H, Schulman AR, Dorbala S. ${ }^{18}$ F-FDG PET/CT for the assessment of myocardial sarcoidosis. Curr Cardiol Rep. 2013;15(4):352.

11. Han EJ, Jang YS, Lee IS, Lee JM, Kang S, Kim HS. Muscular sarcoidosis detected by F-18 FDG PET/CT in a hypercalcemic patient. J Korean Med Sci. 2013;28(9):1399-402.

12. Braun JJ, Kessler R, Constantinesco A, Imperiale A. ${ }^{18} \mathrm{~F}$-FDG PET/CT in sarcoidosis management: review and report of 20 cases. Eur J Nucl Med Mol Imaging. 2008;35(8):1537-43.

13. Kumar A, Dutta R, Kannan U, Kumar R, Khilnani GC, Gupta SD. Evaluation of mediastinal lymph nodes using (18)F-FDG PET-CT scan and its histopathologic correlation. Ann Thorac Med. 2011;6(1):11-6.

14. Huggins JT, Doelken $P$, Sahn SA, King $L$, Judson MA. Pleural effusions in a series of 181 outpatients with sarcoidosis. Chest. 2006;129(6):1599-604.

15. Navaneethan SD, Venkatesh S, Shrivastava R, Mehta J, Israel RJ. Recurrent pleural and pericardial effusions due to sarcoidosis. PLoS Med. 2005:2(3), e63.

16. Salazar A, Mana J, Sala J, Landoni BR, Manresa F. Combined portal and pulmonary hypertension in sarcoidosis. Respiration. 1994;61(2):117-9.

17. Oparka J, Yan TD, Ryan E, Dunning J. Does video-assisted thoracic surgery provide a safe alternative to conventional techniques in patients with limited pulmonary function who are otherwise suitable for lung resection? Interact Cardiovasc Thorac Surg. 2013;17(1):159-62.

18. Roviaro G, Varoli F, Vergani C, Maciocco M, Nucca O, Pagano C. Videoassisted thoracoscopic major pulmonary resections: technical aspects, personal series of 259 patients, and review of the literature. Surg Endosc. 2004;18(11):1551-8. 